\title{
SmartEx: a case study on user profiling and adaptation in exhibition booths
}

\author{
Ben Salem • Jorge Alves Lino $\cdot$ Matthias Rauterberg
}

Received: 24 November 2009/ Accepted: 6 May 2010/Published online: 18 June 2010

(c) The Author(s) 2010. This article is published with open access at Springerlink.com

\begin{abstract}
An investigation into user profiling and adaptation with exhibition booth as a case study is reported. First a review of the field of exhibitions and trade fairs and a summary introduction to adaptation and profiling are given. We then introduce three criteria for the evaluation of exhibition booth: effectiveness, efficiency and affect. Effectiveness is related the amount of information collected, efficiency is a measurement of the time taken to collect the information, and affect is the perception of the experience and the mood booth visitors have during and after their visit. We have selected these criteria to assess adaptive and profiled exhibition booths, we call smart exhibition (SmartEx). The assessment is performed with an experiment with three test conditions (non-profiled/non adaptive, profiled/non-adaptive and profiled adaptive presentations). Results of the experiment are presented along discussion. While there is significant improvements of effectiveness and efficiency between the two-first test conditions, the improvement is not significant for the last test condition, for reasons explained. As for the affect, the results show that it has an under-estimated importance in people minds and that it should be addressed more carefully.
\end{abstract}

Keywords Adaptation to user - Adaptive environment . User profile

B. Salem $(\bowtie) \cdot$ J. Alves Lino $\cdot$ M. Rauterberg

Industrial Design, Eindhoven University of Technology,

Eindhoven, The Netherlands

e-mail: mail@bsalem.info

\section{Introduction}

Adaptive environments are the merger of physical spaces with information and communication technology (ICT) infrastructure. They are combinations of user experience, contents, services delivery, and infrastructure. These environments can be ranked according to adaptation and personalisation. Adaptation is related to adjustments and changes in the service delivery to match user profile to the service provided. It is a change to fit the user (e.g. Currency used in prices to match user location). On the other hand, personalisation is about giving some experience of a service that matches details and characteristics that are not necessary relevant to the service provided or do not make any difference to it (e.g. background music matching personal preferences). It is about ascribing to the service such qualities as private, individual or discretionary.

The adaptation to the users can take many forms and many levels, and in most cases the adaptation is related to the features of the environment and can also be about the services delivered within the environment. We have established five levels of adaptation: reactive, interactive, perceptive, receptive and proactive (see Fig. 1). In parallel, this has an equivalent from the personalisation perspective in the following ranking: Anonymous, Identity, Preferences, Profile and Model.

At the simplest level there are reactive systems that are triggered by user inputs. These are anonymous and they relates to services that do not take into consideration the user particulars (e.g. lift interface). Next come interactive systems that prompt the user and react to his/her actions. At most, these systems take into account the identity of the user (e.g. smart tag for doors control). More complex are perceptive systems, which further to the interactive systems, rely also on implicit user input such as frequently 


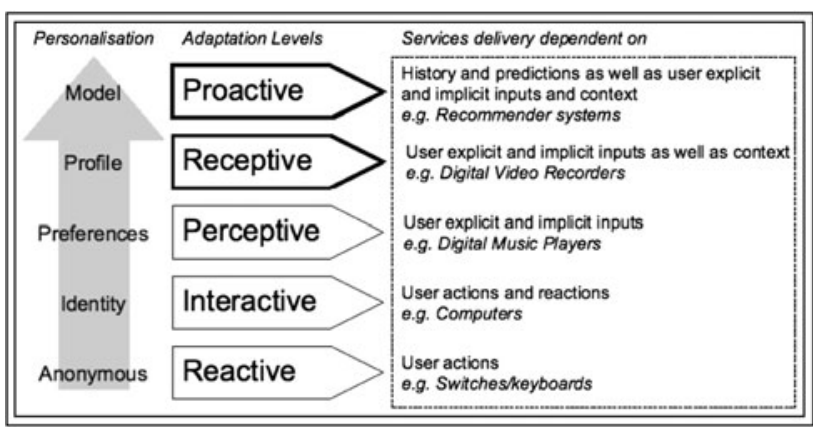

Fig. 1 From reactive to pro-active adaptation

occurring pattern of actions. Perceptive systems are concerned with user preferences and try to deliver services accordingly (e.g. setting preferences in luxury cars). Receptive systems go beyond this and take into account the context of use. Receptive systems take into account user profiles and needs. These systems tailor services delivery according to user profiles (e.g. profiles in online communities). Finally proactive systems are also capable of predicting users actions and preferences according to a user model. It is about setting a representation of the user taking into account his/her profile and history. It also include prediction about user requests and service usage (e.g. some online bookseller recommendations).

\subsection{Adaptation and personalisation}

By clustering adaptive components to deliver services and content and focusing on the user experience, the environment becomes responsive. The responsiveness can be in the form of the physical structure of the space (e.g. movable panels and partition walls). The changes can also be related to the ambient features of the space such as lighting, acoustics and temperature. Finally, the changes can relate to the content presented in the space, like media, information, and interactivity available. Clearly there are many avenues to adaptation and ultimately responsiveness. We believe in the need to build system demonstrators to investigate various content, design, technology and interaction solutions.

Adaptive environments can be implemented in various ways, in our case, we have selected an adaptation based on user profiles and the delivery of accordingly profiled services in the context of a group of users. User profiles were suggested as an improvement for a variety of applications, query enhancement (Korfhage 1984), digital libraries (Amato and Straccia 1999), the personalisation of websites (Goel and Sarkar 2002), enhanced interpersonal communication (Lukose et al. 2003), and in-flight entertainment (Liu and Rauterberg 2007). As for adapted services, current trends are towards the integration of the user profiling in the delivery of services for an aware environment such as customised museum tours (Oppermann 2005), or exhibitions (Kraemer and Schwander 2001).

In adaptive environments where group of users are involved, the issues to address are more complex then in single users cases. Environment adaptation for multiple users is based on (1) the environment awareness of each individual users, (2) the merging, combination of multiple users profiles, (3) the resolution of any conflicts that might occur, shall they be of resources, services, or of interests, (4) the adaptation to the users as a group and as individuals. In fact, the whole group has to be modelled, as for example to select background music for a group of fitness centre users (McCarthy and Anagnost 1998), to recommend a movie to a group according to individual tastes (O'Conner et al. 2001) and in a scenario of selection of television items for a group of viewers (Masthoff 2004).

\subsection{Adaptation strategies and user profiles}

For adaptive systems designed for group of users, several adaptation strategies exist, some strategies deal with service delivery other relate to the management of resources. Stock et al. (2007) propose a voting mechanism in which the highest ranked presentation is displayed. In the case of several visitors interacting simultaneously with the same presentation, they have developed a voting mechanism that relies on a collection of topics of interests for each visitor. Visitors are invited to review the list and vote for one of the topics. The topic with the largest number of votes is then selected and its related presentation shown to the visitors.

Other strategies were described at length by Masthoff (2004) such as "Borda count" where points are awarded to each alternative according to individual preference. In this case the ranking of choices is done by adding individual rankings of that choice to give its group ranking. Another approach is the "fairness strategy" where top items from all individuals are selected. This strategy is based on the belief that individual will accept other's choices as long as theirs are selected. Finally, the "most respected person/ dictatorship" is a strategy where the ratings of the most respected person are used for the selection of alternatives.

The strategies are either based on some user inputs (for example a voting mechanism) or are based on users profiles. Within the scope of this paper we are interested in the adaptation strategies that are based on user profiles. These strategies are the ones that have the potential to deliver automatic adaptation, as they rely on knowledge about the user rather than on observation of the user. Users profiles are about users Needs, Requirements and Desires (NRD). We have determined that NRD of visitors are of paramount importance in the set up of their profiles to create adaptive and interactive experiences (Salem and Rauterberg 2004). 
The visitor profiles can be set up implicitly (or as a reaction) from visitor history (i.e. past visits), or explicitly in advance, via registration, before the booth visit. Explicit profiles are however more useful in the context of this project as they are an already prevailing practice. Furthermore. we are interested in strategies that are applicable to group of users and where there is a conflict of resources. There could be a conflict when there are limited resources, or when there are contradictory users NRD, which are competing with the available resources. In this context there has been many studies for the evaluation of various strategies. However most have been questionnaire based (notably (Masthoff 2004)). While these studies yield interesting results they are attitude based (in the sense that they rely on the responders attitudes). We would like to conduct an evaluation that is behaviour based (in the sense of the evaluation being based on actual behaviour of the responders)

\section{Trade shows and exhibition booths}

We have investigated which field could be used as a case study for our investigation of adaptive environments and at the same could benefit from our work. In this perspective, the world of trade shows and exhibitions is one with a lengthy history. Exhibitions include a variety of events, from small and local such as trading fairs, all the way to the World Expos. In a modern exhibitions, we find a similar arrangement across the range. On the one hand the exhibitor is in a booth, advertising about her/his products or services through a variety of media, trying to attract as many "target" visitors as possible. On the other hand the visitors are wandering between the booths, looking for something that will attract their attention and meet their interests. Trade shows are an opportunity for companies to meet new customers, launch new products and assess the market. For visitors, the main reasons invoked to attend are the desire to see particular products or companies, followed by information gathering such as product, technical and industry information (Rosson and Rolf Seringhaus 1995). Due to the variety and the number of visitors in a trade show, exhibitors do not always have the opportunity to effectively interact with all the visitors. It is also known that booth staff have difficulties in adapting to a variety of visitors and a range of requirements and needs (Bello and Lohtia 1993). There is most often, such a strong a focus on sales that exhibitors are required to pay attention (sell) to visitors who participate in buying decisions and to ignore and avoid focusing on those without buying authority (Bello and Lohtia 1993). However, it is important to avoid too much an emphasis on sales as it often turns out counterproductive (Tanner and Chonko 1995).
Innovations in the world of exhibitions are not easily accepted and mostly focus on changes in the way media are used or information presented, but there is some acknowledgment in the professional literature of the promising perspective of adaptation in exhibition booths, [notably (Bello 1992; Rosson and Rolf Seringhaus 1995)]. There is also acceptance that information presented should match visitor interests and be concise (Sashi and Peretty 1992).The information displayed and distributed in exhibition booths should focus on the visitor needs and interests. It is important to address the different needs and information requests of the variety of booth visitors, otherwise visitors might develop misunderstandings about the information they are gathering (Tanner and Chonko 1995). Furthermore, there is also some recognition of the potential for prioritisation and organisation of information in booths (Banting and Blenkhorn 1974; Bello 1992; Munuera and Ruiz 1999).

We have concluded that trade exhibitions were suitable and in need of new developments in the form that we propose: an Adaptive Exhibition Booth (AEB) we call SmartEx (for Smart Exhibition). SmartEx combines an infrastructure that process and display information about an exhibit in an adaptive manner in relation to the booth visitors. We see the AEB as a system that matches information and contents to booth visitors. The AEB allows for the personalisation of booth visits, it is achieved in a similar fashion to recommender systems that match products to buyers (Wang et al. 2004). In the service industry, when important buying decision are involved, quality, customisation and friendliness are more important than price (Ostrom and Iacobucci 1995).

\subsection{Exhibition booth assessment}

Whatever innovation is proposed, some assessment of its beneficial contribution to the world of trade fairs is essential. To evaluate the effectiveness of an exhibition booth, Cavanaugh (1976) relies on both quantitative and qualitative data. The first type of data is made up of the head count of target audience, number of sale leads, ratio of projected vs actual target audience, ratio of total cost vs total number of target visitors. As for the qualitative data, it is the visitors reaction (in purchasing terms) to the exhibition booth and meetings with staff, the average time spent in the booth and the competitors activities in at the show. This is however does not take into account the visitor experience and the appreciation or lack thereof they might have of their visit. To assess a trade show, Bellizzi and Lipps (1984) talk about audience quality, audience activity and exhibit effectiveness. They define audience quality as a measure of the total buying plans, or as the percentage of the audience who are buying a product they saw at the 
exhibition. The audience activity is mostly related to the duration of an exhibit visit, and the number of exhibition booths visited. As for the exhibit effectiveness, they define it as how well did the exhibit attract prospective buyers and how well did the booth staff establish personal contact with the booth visitors. These measurements where expanded by Sashi and Peretty (1992), who added for the exhibit performance the product interest, the buying influence, the buying plan, the memorability and the cost per visitor reached. These relate respectively to the percentage of visitors interested in the exhibitor product(s), the percentage of visitor with a buying influence, the percentage of visitors with a plan to buy a product within 12 months, the percentage of visitor who remember visiting after $8-10$ weeks, and finally the ratio of the cost of exhibiting by the number of visitors. Herbig et al. (1994), reuse these parameters and narrow them. Audience quality indicators become purchasing power, audience activity indicators are average time spent in a booth, and exhibit effectiveness indicators are narrowed down to cost per visitor and sale generated at show. These are however one sided parameters focusing on the exhibitor interests and not those of the visitors. The evaluation of an exhibition booth can be measured differently; by assessing the performance of the selling (actual purchases, orders placement...) and the nonselling functions (product promotion, demonstrations...) of the booth (Kerin and Cron 1987). However, the performance of a booth means different things depending on whom is assessing the booth. On a different approach, Williams et al. (1993) talk about booth attraction and booth contact. Booth attraction is the ratio of the number of interested visitors who attended the booth over the number of interested visitors at the show. The size and location of the booth, the products displayed and demonstrated, the pre-show promotion(s) play all a role in the booth attraction. As for the booth contact, it is the ratio of the number of interested visitors who attended the booth and were contacted by the booth staff over the number of interested visitors who attended the booth. The number of booth staff, their background and training play a role in the booth contact.

Booth visitor experiences and interests are not given much importance it seems. There is too much a focus on exhibitors interests and objectives. This is problematic, and the industry-wide knowledge states clearly: It is always easy to get exhibitors to a trade fair but much more difficult to get visitors. During our field enquiry, we have observed current practices used by exhibitors to maximise encounters with visitors, they fall into 3 groups: (1) pre-arrangement, (2) identification and (3) initial greeting. In the first group are the pre-arranged visits with appointments and set times for discussions. In the second category, the identification of the various actors within a trade show is highlighted. Typically, each of the Exhibitors, visitors and VIPs wear a different badge (colour, pattern, shape, etc.). There is a strong visitors dislike for the identification scheme as reported to us. The third group is about the initial greetings filter. When entering a booth one is confronted with direct questions about one's background, generally broken up into three questions: position, role and company. If one does not give the right answers, one is ignored right away, a clear indication that some visitor profiling occurs in an exhibition.

It seems also that priority is given to top profiled people while others are ignored. This can be compared to the adaptation technique called "Most Respected Person Strategy", also known as "dictatorship" as described by Masthoff (2004). Compared to other selection strategies this one seems rather simple but is closest to current industry's practices. This strategy is in essence a combination of identification of the participants and prioritisation. In an AEB setting this would result in the top priority profiles acting as the main user of the environment. This profile will be controlling the selection of presentations running and contents available in the booth, lower priority profiles would follow in a decreasing order of priority. The lowest priority profile person at the end of the scale will only be able to watch his/her presentation when no higher profile is present. Although this is a rather simple adaptation technique, we have selected it as it is the current industry practice.

\subsection{Selecting assessment criteria}

When a company has well defined market objectives, trade shows evaluation and assessment are easier to perform as the metric to use are clearer. Unfortunately, many companies do not specify the objectives of their participation to a trade show let alone assess it (Gopalakrishna and Lilien 1995).

We have collaborated with the Dutch market leader in exhibitions, a company called Gielissen. It is a vertically integrated company that designs and builds exhibition booths, is a main subcontractor to exhibitions and helps exhibitors define their strategies. We have also called upon the assistance of PLMA (Private Label Manufacturer Association, private labels are companies that manufacture products for other companies to sell). PLMA organises one of the largest trade fair (named PLMA) in Europe. Our industrial partners, gave us the opportunity to attend several exhibitions and observe what it is to be an exhibitor and experience first hand what it is to be a trade show visitor. After further discussions with our industrial partners, we have concluded that from an exhibitor point of view efficiency and effectiveness of the exhibition is of great importance (as already highlighted in the review 
section). How much information (in this case we are talking about information that leads to the exhibition objectives such as sales, marketing...) is gathered and how long does it take to do so are the two key focuses for the exhibitors. From a visitor perspective time is very important, in general visits to a booth should last no more than 15-20 min, visitors will walk at fast pace between booths to minimise time spent and will require information (I this case we are talking about information that matches visitors NRD) to be presented at them rather than have to seek it. Information-push and time-saving appear to be the preferred approaches.

As a result of our own observations, discussions with our industrial partners and the field review, we have adopted effectiveness and efficiency as key criteria for the assessment of AEB success. We investigated these two issues and found out that exhibitors consider effectiveness as an indication of the amount of information gathered. Exhibitors relate efficiency to the time taken to visit an exhibition booth. Exhibitors consider that there is enough information gathered when visitors could answer a series of questions about the exhibitor and its products or services. Visit time is related to the time taken from entering the booth until leaving is.

Regarding the amount of information gathered, we have included questions about the exhibitor and its products or services as part of our experiment questionnaire described in Sect. 4.8. As for how long does it take to visit the exhibition, we have measured the time taken and looked for a reduction of it between different test conditions to highlight the most efficient set-up.

However efficiency and effectiveness are not enough for a successful AEB. We also discovered that the affect of an exhibition visit was of great importance to both exhibitors and visitors. For the exhibitor, it mattered because it influenced the perception the visitor had of them. For the visitor it qualified the experience they had. We decided to add a third assessment criteria alongside effectiveness and efficiency, namely affect, the subjective experience resulting from the visit and its effect on the visitor moods, emotions and attitude towards the exhibition and the exhibitor. We also wanted to verify if adaptation would have any affect on the booth visitors. In this perspective, we propose to re-organise the information presented to visitors within a usual exhibition booth. In our case an exhibition booth about Gielissen that we set up in our department.

\subsection{Setting-up profiles}

The visitors of an exhibition can be: (1) genuine visitors with buying/decision-making power, such as commercial directors or purchase managers; (2) genuine visitors without power, such as low ranking employee or students; (3) competitors acting as visitors who are enquiring about the current state of the art; (4) "sightseers" who are visiting either for reasons outside the scope of the trade show, like booth designers investigating the latest trends; or are visiting for non-business reasons such as meeting friends who are exhibiting.

In this area, Hoshen (1989), has identified four visitor profiles: (1) interested visitors without purchasing intentions, (2) potential purchasers, (3) actual purchasers and, (4) other visitors (such as salesmen). Similarly Spiegel (1992) has reported four profiles of visitors: (1) Intensive User (full and frequent visitor to Exhibitions), (2) special purpose user (intending on purchase decision), (3) stroller (observing the market) and (4) pragmatist (seeking technical information).

As for Bello (1992), he has used five profiles that can be amalgamated into three ordered profiles: (1) Senior management, (2) policy level, (3) operating level.

Accordingly, and in co-operation with our industrial partners we have set-up four visitor profiles, in order of precedence:

1. Senior manager with purchase potential (e.g. division director),

2. Policy level manager with no purchasing but selling intentions (e.g. marketing director),

3. Operating level staff, actual purchaser (e.g. technical director),

4. Operating level staff without purchasing intentions (e.g. Design director).

User profiles are made of an explicit identification of users and a listing of their particulars (demographics, function and professional background...). Users profiles are also about users NRD- Needs (accessible booth and information, understandable content,...), Requirements (matching of content with interest in line with their particulars..) and Desires (content preferences, and matching of content with secondary personal interests...), within the context of the services provided and the contents available.

\subsection{Related projects}

There are many projects that are related or of relevance to adaptive environments. Interactive environments are a notable example; they are spaces that interact with their visitors. They connect art, technology and interactive installations (Bullivant 2006). These environments are at the forefront of technology and have been playing groundbreaking roles since the 1970s with the work of interactive artists [see for example (Krueger 1983)].

In the area of trade shows, the mobile Fairguide is a system implemented during CeBIT 2001 that delivers 
services related to location-dependent information, personal profiles and database information (Kraemer and Schwander 2001). Fairguide was set up using Bluetooth technology, which due to its limited range, can also act as a tracking system (in this instance the resolution was 10$20 \mathrm{~m}$ ). The systems provided Internet access, information distribution and ticker-tape broadcast. The main role of the mobile Fairguide was to act as support to the exhibition and as an extension of its information channels. Another system, the mEXPRESS was developed as a wireless exhibition guide by (Pateli et al. 2004). The system gives guidance and information to the visitors, locates people in the exhibition venue and can deliver promotional material. It delivers three kinds of services: information, navigation and communication. The system is rather pro-active in the delivery of services, it alerts the visitor about exhibitors matching his/her interests, delivers advertisements and common announcements, the system is also used for postshow marketing (using recorded visitor behaviour). eGUIDE used infrared technology for communication between broadcast cell and hand held devices. eGUIDE provided a set of services for visitors such as finding directions, information about exhibits, planning of personal tours, appointments and navigation within the exhibitions. Other information such as cash points and restaurants was also part of the service. On the other side, the exhibitors received up to date data regarding the number of visitors in a specific time-interval as well as their location within the exhibition (Bieber and Giersich 2001). Other studies have used personal digital assistants (PDA) or handheld computers as a basis for augmenting the physical social space (McCarthy and Anagnost 1998). One advantage of using a Personal Digital Assistant (PDA) is that it provides a personal display. For example, the SpotMe Conference Navigator $^{1}$ is a conference matchmaker that runs on a PDA using RF to perform one-on-one profile matching, notifying visitors with similar profiles when close by. Unfortunately, there is no user study reported on the use of this device.

In the related field of museums, the HIPPIE guide provided a multimodal information presentation of the art collection (Oppermann and Specht 1999). The information about the exhibits was composed according to visitor preferences. The system could be used for preparation of the visit, during the visit and as a visit memo. The mapping of the user interests was based on the taxonomy of all exhibits according to types (e.g. paintings, sculptures, art crafts...) and genres of artwork (e.g. mythology, religious, portrays...) and on taxonomy of attributes (e.g. biography, history, motives...). The evaluation results showed that the adaptive features of the museum guide were considered

\footnotetext{
${ }^{1}$ See: http://www.spotme.ch
}

helpful. In another project, PEACH, an adaptive intelligent presentation of information is proposed, as part of a museum visit by Stock et al. (2007). Some of the aims of this project are similar to ours. User modelling was the basis of the personalisation of content. Multimedia presentations that match user models were used to enrich the museum visit. For the ubiNEXT project, an educational museum PDA guide was used to enhance museum visitor's learning and to support the entire museum experience (Bartneck et al. 2007). The system combine audio and visual presentations about a museum exhibits. The museum provides visitors with Internet and on-site services. The Internet service has two major features: a pre-visit planning and a visit review. At the museum a PDA presents object information and educational activities with interactive test, audio and videos. Exhibit locations are also presented.

In the three museum projects, adaptation can be understood as location or profile dependent information, presented on a personal device. The use of personal displays such as PDAs, rather than the booth infrastructure has serious limitations. The exhibition staff is not aware of what information the visitor is currently being exposed to and thus cannot seamlessly engage with her/him. Exhibitors will have to rely solely on requests from visitors for further information; this would limit marketing and sales opportunities.

\section{Objectives}

We would like to assess if AEB yield better exhibitions and if visitor profiling and adaptation will yield a more effective, efficient and positive experience of the otherwise tiring exhibition visit (as reported to us by exhibition visitors). The aim being to investigate if an adaptive trade show can yield significant advantages over a conventional exhibition. We propose to run an empirical experiment about AEB by comparing them to conventional exhibition booths.

After reviewing related work and projects, we have come up with the conclusion that there is a need for empirical studies yielding behaviour-based results. We have decided to set up an installation of an exhibition booth as close as possible to the real world and run empirical testing within that installation. We would like to know what are the benefits if any of an adaptive exhibition in a realistic setting.

\subsection{Detailed objectives}

We propose to assess if AEB are more effective and more efficient than conventional exhibition booths. The validity of these two criteria have been confirmed during our field 
enquiry. We need to investigate if profiled presentations yield a more effective, efficient exhibition with better visitors affect. A profiled presentation is a presentation that has been edited to contain only information relevant to a particular visitor profile. It is in a way a compiled and shortened presentation that includes only the relevant information. As such, profiles presentations should be more effective as source of information and more efficient. They should also yield a better affect on the booth visitors by giving them the opportunity to gather information faster and easier. Furthermore, we would like to know if adaptive profiled presentations yield a more effective (i.e. acceptable amount of information collected), more efficient (i.e. minimal time spent) exhibition with better visitors affect. An adaptive exhibition booth displays profiled information according to the implemented adaptation strategy. It is an automatic presentations selection and should be more effective and more efficient than non-adaptive exhibitions. It should also have a better affect on the exhibition visitors.

ISO 9241-11 has the following definitions for effectiveness, efficiency and satisfaction:

- effectiveness: Accuracy and completeness with which users achieve specified goals.

- efficiency: Resources expended in relation to the accuracy and completeness with which users achieve goals.

- satisfaction: Freedom from discomfort, and positive attitudes towards the use of the product.

In the context of our paper, the effectiveness of the AEB can be measured in terms of the amount of relevant information gathered from a visit to the booth. The relevance of the information collected is tailored to the visitor NRD and not the exhibitor objectives. At the same time, the efficiency of the AEB can be measured as being the amount of time required for an effective visit of a booth. We would also like to take into account the affect the AEB has on the visitor experience and mood. We can therefore summarise our aims as being about investigating an AEB and assessing whether it delivers a more effective, more efficient exhibition and has a better affect on the exhibition visitors.

\section{Method}

We have set up a conventional exhibition booth made of two parts, a richly decorated section (Fig. 2) and a simple section for meetings (Fig. 3). Within the booth we have set up three displays in three viewing areas. In previous exhibitions these displays would present a slide presentation about the exhibitor. We edited the presentation that has been previously used at an exhibition by our partner

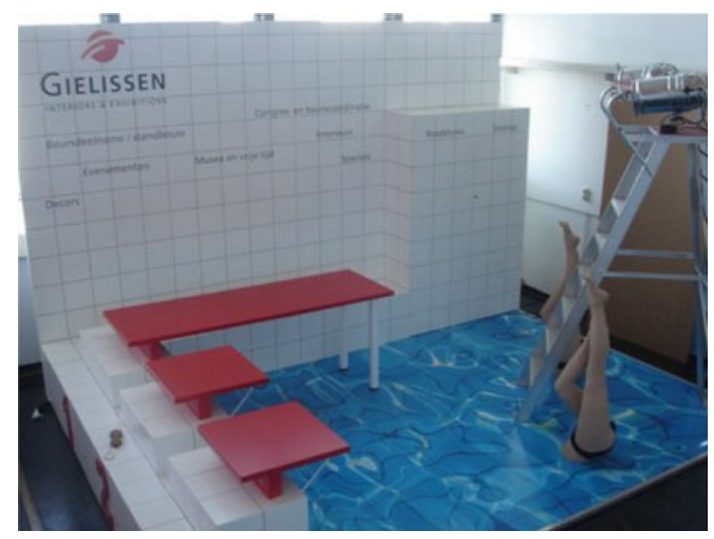

Fig. 2 Standard booth (showing viewing area 2)

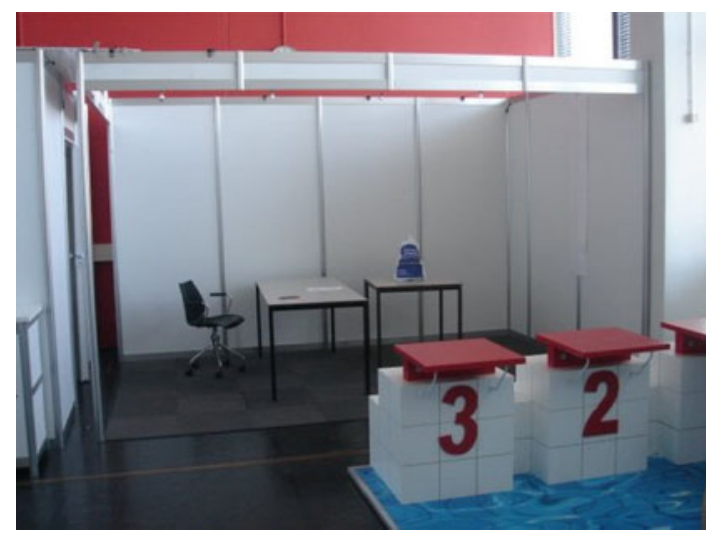

Fig. 3 Standard booth (showing viewing area 1)

Gielissen. We would like to end up with a set of presentations that are customised for each identified profile of booth visitors. We group the visitors into profiles (set up according to our industrial partner recommendations). We use these profiles to identify the NRD of the visitors and to match the setting of presentations shown accordingly. We therefore created different presentation versions that are of particular interest to the visitors of different profiles. In essence, we are proposing AEBs that delivers adapted personalised information based on user profiles [e.g. (Fan et al. 2005)].

Three displays were set up in the booth, in the three viewing areas, showing different slide presentations (depending on the test conditions, see layout in Fig. 4). Distributed in the booth were the usual goodies and freebies such as pen, golf balls and sweets with the exhibitor logo. There also were various leaflets about the exhibitors but these contained only very general information about the company of no relevance to the tasks participants had to perform. The exhibitor (booth staff) was standing by the chair visible on Fig. 3. 


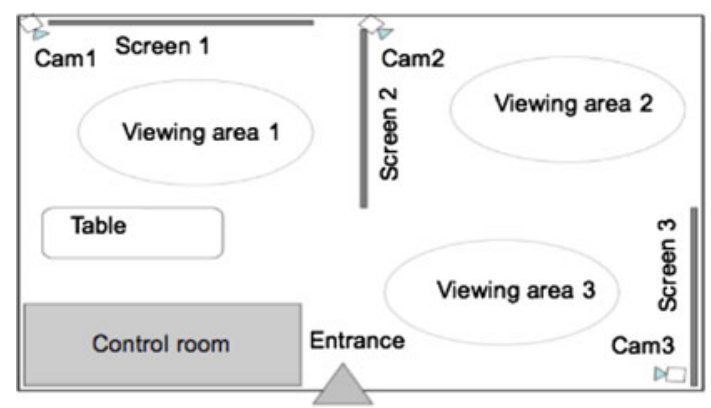

Fig. 4 Layout of the booth (with three viewing areas)

\subsection{System description}

The set-up was an exhibition booth kindly provided by Gielissen. The overall layout of the booth is shown in Fig. 4.

\subsection{User profiles}

We have established for our visitors 4 ordered profiles. These were, in order of precedence:

1. Division director, with the task of finding out if the exhibitor is a viable partner for long-term business.

2. Marketing director, with the task of finding out if the exhibitor could become a client to his/her company.

3. Technical director, with the task of finding out what kind of contractor the exhibitor is.

4. Design director, with the task of finding out what is the exhibitor design style.

It is important to note that the profile distribution in the visitor population might have a detrimental effect on the experiment results, this why we believe it is very important to have a realistic distribution that is a true reflection of real trade fairs. The challenge is to ensure the right granularity and completeness. Profiles 1,3 and 4 have the role of a "buyer", while profile 2 is a "seller". As a buyer one is looking for information about the exhibitor and assessing if they could match his/her needs. As a seller a visitor is trying to assess if the exhibitor could become a client to their company services. During the experiments, all the visitors were regrouped into three companies, a start-up (3 of each profiles), a SME (Small/Medium Enterprise) (3 of each profiles but no design directors) and a PLC (Public Limited Company, a large company listed on the stock market) (three division and design directors).

We assume also that user preferences do not change during the visit. As our profiles are based on individual professions and roles in a company, these are unlikely to change during a visit, however there is nothing to prevent a CEO of a company to have personal interest in design issues that $\mathrm{s} / \mathrm{he}$ would like to know more about (in which case $\mathrm{s} / \mathrm{he}$ can always ask for and seek more information then required in the experiment pack).

\subsection{Participants}

Participants were all students of the Eindhoven University of Technology, either enrolled in the bachelor of the Department of Industrial Design or in the Post-Master of the User System Interaction program. Both educational programs are in English so all students are fluent in the language. We had 27 participants of which 13 were females and 14 were males. $52 \%$ of the participants were in the age group 25-30, the reminder evenly distributed between the 20-24 and 30-35 age groups. Although the participants were not experienced exhibition visitors, they were given clear instructions (via verbal explanations and the experiment packs) as to what tasks they would be facing.

We also ensured that people profiles were not displayed during the experiment and used instead a differently coloured piece of cloth for identification purposes, during all the test conditions. All the participants were required to don the pieces of cloth whose colour matched their profile (visitors were only instructed to wear the pieces of cloth with no further explanations).

\subsection{Tasks description}

For our experiment, participants were requested to enter a simulated exhibition booth and to perform a task related to their profiles. They were set to be the closest possible to real tasks performed by exhibition visitors. Tasks were essentially about finding information about what Gielissen as an exhibitor had to offer in terms of services. In one case however, the profile of marketing director, the task was to find out if Gielissen could become a client to the visitor's company. They had to enquire about the exhibitor and answer a series of questions contained in the experiment pack handed to them (see Sect. 4.7). Participants were told to be comfortable and behave the way they would in an ordinary trade show, visiting an exhibition booth. If at any point they had questions about the exhibition itself, the company or the products displayed, participants were told to not hesitate to ask questions to the exhibitor, as they would if they were in a real exhibition. Participants were also told they will not be alone in the exhibition and each of the participants in the experiments will have a task to perform in the form of a questionnaire to answer. Thereafter they were asked to bear in mind their task and ensure they performed it as they saw fit. Furthermore, they were instructed to visit the booth at a normal pace but without wasting time, as the duration of their visit will be measured. 
Participants will be given a profile and during the tests a questionnaire with a series of questions ranging regarding the exhibitor and some products shown at the booth. Questions will be closely matching visitor profile. It is important to note that visitors will share same tasks if they share same profile (e.g. a buyer is always interested in price and availability of product...).

\subsection{Adaptation technique}

We have selected the 'Most Respected Strategy' for our system adaptation, as it is the strategy most closely matching current practices by exhibitors. During the adaptive test condition (profiled adaptive presentations), the booth's presentations running concurrently on three displays are selected according to the highest priority visitor who is watching. We would also like to ensure that each individual satisfaction with the exhibition should be above a certain threshold. There should be no visitor that finds no useful information whatsoever. This is why we have ensured that as soon as high priority visitors have fulfilled their tasks, and left the viewing area, next priority visitors are then attended to with their corresponding presentation being displayed. There should also be no visitor that needs more that $20 \mathrm{~min}$ to find a minimum of information to answer the questionnaires participants were given. A wizard of $\mathrm{Oz}$ setting allows the selection of the presentations for the third test condition (profiled adaptive presentations). We had three volunteer operators, hidden in an enclosure controlling the three personal computers driving the three data projectors. While participants in the experiment would wander in the exhibition booth, video camera would let the three operators know who was watching which display. The operators would switch between presentations according to the highest priority of those watching the screen, as soon as they looked at the screen.

\subsection{Role of booth staff}

The exhibition booth was staffed in all the test conditions. The exhibitor role was similar to those in real trade shows. He was instructed to normally engage in conversation with visitors and to answer their questions if they insisted on. The rules to be followed were inspired from the adaptation strategy selected. The exhibitor will engage with and answer questions of the visitor whose ranking was the highest first. The exhibitor was instructed to answer questions by order of priority, and to cut short a conversation if someone of a higher priority approached. We were hesitant to staff the exhibition booth but at the end we decided to have our set up as close to a real exhibition booth as possible.

\subsection{Experimental set-up}

We propose to run an experiment with various test conditions to measure the differences if any the adaptation and the user profiles may have. The test conditions correspond to the various configurations we have set up. All test conditions are run in the same exhibition booth installation that has displays and viewing areas. Visitors are asked to enter the exhibition and perform tasks we have given them (see Fig. 5). We measure the time duration of their visits and we assess their performance of the tasks given via questionnaires. As our intention was to reproduce an as realistic as possible exhibition booth, the experimental setup was not totally controlled and participants had some freedom regarding their behaviour. For instance they were allowed to wander around the booth, to approach the exhibitor and to talk to other participants (although none did in a way that was detrimental to the experiment).

Within the same exhibition booth and keeping all conditions the same, we ran the experiment with three independent variables: (1) test conditions as between subjects; (2) user profiles as between subjects; (3) tasks as within subjects. There were three test conditions, four visitor profiles, and 27 participants. The three test conditions are: (1) non-profile, non-adaptive presentation; (2) profiled, non-adaptive presentation and; (3) profiled, adaptive presentation.

\subsubsection{Test condition 1: non-profiled, non-adaptive installation}

A single presentation containing all the information necessary for all the profiles is presented running in parallel loops on the three displays in the booth. The three presentations have a different starting time.

In our experiment, the non-profiled presentation includes all the information relevant to all the visitors. It forms the basis of all the other presentations.

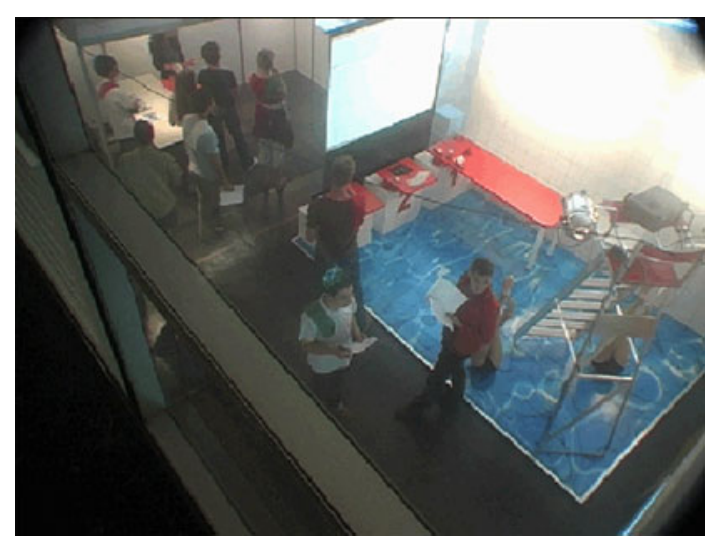

Fig. 5 Participants during the experiment 
Visitors have to wander around in the booth and look at the presentations until they have gathered all the information necessary to complete their tasks. There is neither adaptation nor changes in the presentations displayed.

\subsubsection{Test condition 2: profiled, non-adaptive installation}

Four profiled presentations run one after the other in three loops displayed on the three displays of the booth. The three loops have a different starting time.

A profiled presentation is a shorter version of the nonprofiled presentation that has been edited to contain mostly information relevant to a particular profile. We have created four profiled presentations matching our profiles. Once again the visitors have to wander around and there is no adaptation or changes in the presentations displayed.

\subsubsection{Test condition 3: profiled, adaptive installation}

In the last test condition, each of the three displays shows the profiled presentations according to the highest priority visitor who is currently watching each display.

The Presentations are displayed according to the visitor who is watching the displays and has the highest priority profile amongst those watching. Changes are made in a Wizard of $\mathrm{Oz}$ setting by hidden operators.

\subsection{Experiment packs}

Participants were given at different times experiment packs written in English. We included in the packs some background facts about the companies each participant would belong to. These packs were:

1. A Pre-test pack: a general introduction to the project, a question asking if the participant would like to take part in the experiment, a questionnaire about background information: personal information, shopping habits, familiarity with retail industry, familiarity with exhibitions, and familiarity with Gielissen.

2. A Test pack: A Description of tasks to perform (essentially to wander around in the exhibition booth and collect answers to a questionnaire), some remarks on how to fill the questionnaire, a bi-polar scaled mood questionnaire, an introduction to the participant company that s/he belongs to, the Description of the participant profile, the set of questions (between 11 and 13) the participant has to answer in line with his/ her profile. This is to assess how much information s/ he has gathered from the visit to the exhibition booth.

3. A Post-test pack: some remarks about how to fill the questionnaire, a bi-polar scaled mood questionnaire, questions about the perception of the exhibition area, perception of the experiment, familiarity with Gielissen, and the performance of the tasks.

\subsection{Measurements}

We have collected two types of data: objective data and subjective data. The objective data measure time duration and number of correct answers. The time duration correspond to the time taken by the visitor to visit the booth and answer all the questions of the participant packs. The number of correct answers counts the total number of answers given in the experiment pack that are correct. The subjective data relate to participant moods and their experience, before, during and after the experiments. Subjective data were collected with a bi-polar questionnaire, self-reporting and general discussions with participants at the end of each test conditions. Subjective data relate the perceived experience visitors had while in the exhibition booth.

\section{Results}

We present these results of our experiments in this section. In all this section horizontal bars in the graphs indicate standard deviation ( \pm std).

\subsection{Overall objective measurements}

In comparing the three test conditions, the estimated mean of task duration falls from $23.00 \mathrm{~min}(\mathrm{std} .=4.77, \mathrm{~N}=9$ ) to $16.89 \mathrm{~min}$ ( $\mathrm{std}$. $=3.37, N=9$ ) and then to $15.89 \mathrm{~min}$ (std. $=3.33, N=9$ ) (see Fig. 6). This indicates an increase in efficiency from a conventional exhibition booth to a profile based one. The same is also somewhat true between a profile based exhibition and a profiled adaptive exhibition. However while the decrease from 23.00 to $16.89 \mathrm{~min}$ is significant (sig. $p=0.010$ ), the decrease between 16.89 and $15.89 \mathrm{~min}$ is not (sig. $p=0.862$ ).

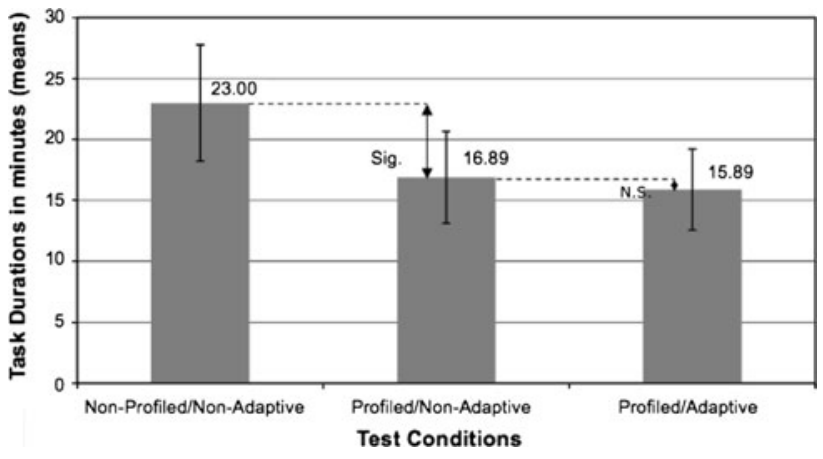

Fig. 6 Mean task duration 
These results are further confirmed with the post-hoc Scheffe test. The mean task duration differences between the 1 st and 2 nd and between the 1st and 3rd test conditions are significant. Respectively, these differences are: $6.11 \mathrm{~min}$ (std. $=1.34$, sig. $p=0.002$ ) and $7.11 \mathrm{~min}$ (std. $=1.34$, sig. $p=0.001$ ). At the same time, the mean task difference between the 2nd and the 3rd test conditions 1 min (std. $=1.34$, sig. $p=0.763$ ) is not significant.

We have also found out that the mean of total number of correct answers has increased from 11.33 (std. $=1.87$, $N=9$ ) to 15.56 (std. $=3.54, N=9$ ) (see Fig. 7). Bearing in mind that all the information presented is identical but organised differently between the test conditions, this is further evidence of the advantage of profiled presentations. There is also an increase of 15.56 to 16.00 (std. $=4.06$, $N=9$ ) when adaptation is added. Again, while the first increase to 15.56 is significant (sig. $p=0.040$ ) the second one from 15.56 to 16.00 is not (sig. $p=0.960$ ).

These results are again confirmed with the post-hoc Scheffe test. The mean number of correct answers differences between the 1st and 2nd and between the 1st and 3rd test conditions are significant. Respectively, these differences are: -4.22 (std. $=1.55$, sig. $p=0.040$ ) and -4.67 (std. $=1.55$, sig. $p=0.022$ ). At the same time, the mean task difference between the 2 nd and the 3rd test conditions -0.44 (std. $=1.55$, sig. $p=0.960$ ) is not significant. Both sets of results show the advantage of profiled presentation. They also indicate that making adaptive presentations successful needs further investigation.

\subsection{Objective measurements per profile}

Looking at the outcome from the perspective of the various participant profiles, the data show interesting results (see Fig. 8). In regard to efficiency, the mean of task duration is decreasing for the two top profiles, i.e. division and marketing directors: from 26.00 to 18.00 and then $12.67 \mathrm{~min}$, and from 22.50 to 18.50 and then $17.50 \mathrm{~min}$. Furthermore the top profile has the largest duration reduction. For the

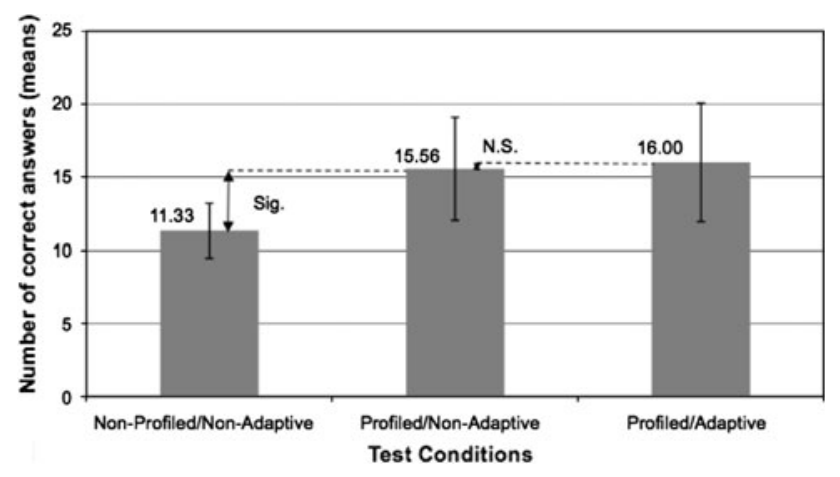

Fig. 7 Mean number of correct answers

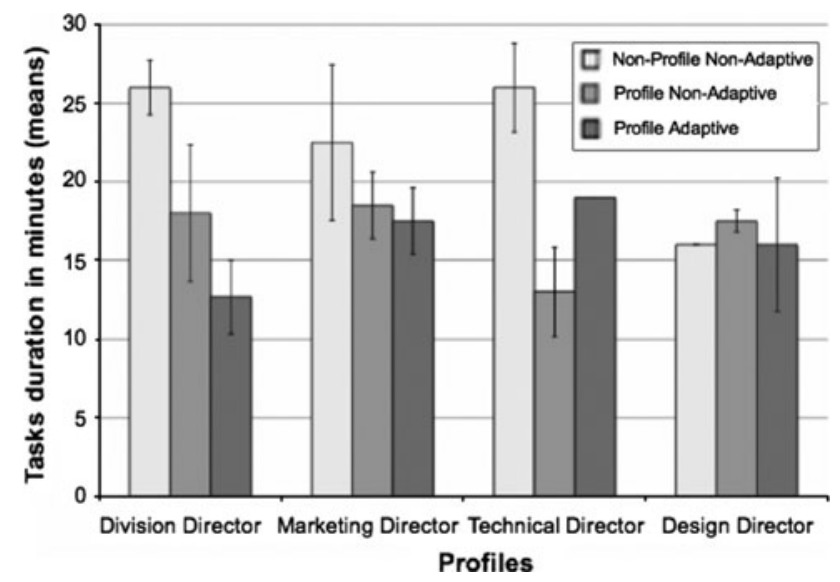

Fig. 8 Task duration per profile

two profiles with lower priority, the results are somewhat different. The technical director (3rd priority) has a decrease of task duration and then an increase, between the three test conditions (respectively 26.00, 13.00, $19.00 \mathrm{~min}$ ). However as a whole this profile performs better in the last two test conditions profile/non-adaptive and profile/adaptive than in the non-profile/non-adaptive. Only the design director (lowest priority) has no change between the first and last test conditions, with both task durations being $16.00 \mathrm{~min}$. The profile is also the only one with an actual increase between non-profile/non-adaptive and profile/non-adaptive conditions (from 16.00 to $17.50 \mathrm{~min}$ ).

Once again similar results are obtained when looking at the number of correct answers (see Fig. 9). For the top profile the improvement is the most important (from 9.67 to 17.33 and then $20.33 \mathrm{~min}$ ). However for the remainder of the profiles the results are not as positive from the perspective of AEB. For example for the marketing director (second top profile) the number of correct answers is somewhat declining between the three test conditions (13.00, 13.50 and $12.50 \mathrm{~min})$. Furthermore, apart from the

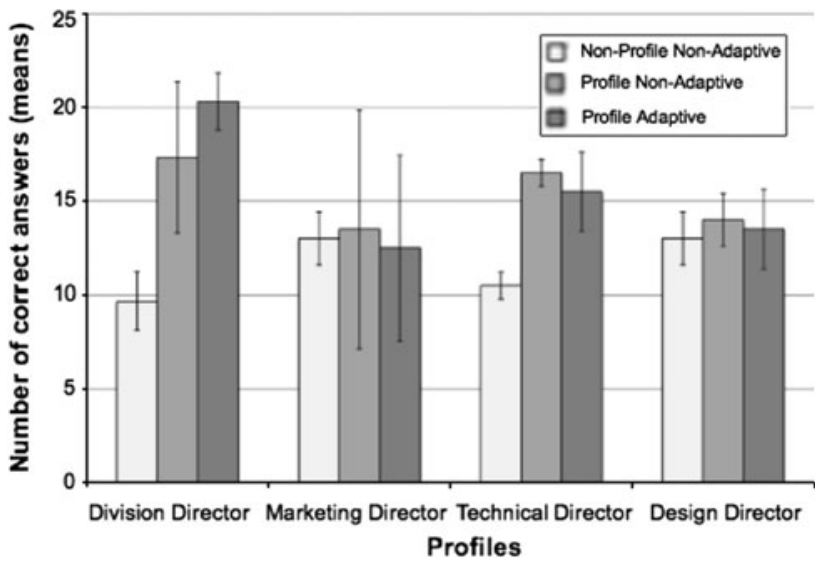

Fig. 9 Number of correct answers per profile 
top profile all the results are actually better from the nonprofile/non-adaptive condition to the profile/non-adaptive condition than to the last condition (profile/adaptive).

\subsection{Subjective measurements}

As part of the test pack and the post-test pack, we handed out a bipolar-scaled mood questionnaire (scaled $-2,-1,0$, 1,2 , and a "don't know").

We asked the following questions:

- What is your mood at the present?

- Tired-Energetic or Don't know.

- Stress-Relief or Don't know.

- Depressed-Cheerful or Don't know.

- Tensed-Relaxed or Don't know.

- In control-controlled or Don't know.

- Focused-Distracted or Don't know.

The results of this questionnaire were not statistically significant, there is a general improvement tendency between the subjects moods as reported before and after each experiment. There is also some variety in terms of the magnitude of the improvement between the three test conditions, but it is only indicated as a tendency (Table 1).

During post experiment discussions (we organised the discussions after the experiments took part with three separate groups corresponding to the 3 test conditions) with the participants, it was made clear that their moods during the experiments were of great importance for them. Most of the comments made by the participants, related to their experience. In particular, those who were given a low priority profile expressed frustrations and also some feeling of unfairness in the cases of the profiled presentations (test conditions 2 and 3). There were also impressions aired that others had easy or shorter tasks. Further discussion indicated that those at low priority were sensitive to the fact that it seemed some of the participants "had it easy" (as said by one of the participants with agreement from all the low priority participants) and could gather all the information they needed, while their presentations seemed to "jump into things not interesting before coming back" to

Table 1 Results from subjective questionnaires

\begin{tabular}{lccl}
\hline Moods & Condition 1 & Condition 2 & Condition 3 \\
\hline Tired-Energised & 0.111 & 0.208 & 0.330 \\
Stressed-Relieved & 0.888 & 0.000 & 1.111 \\
Depressed-Cheerful & -0.222 & 0.000 & 0.111 \\
Tensed-Relaxed & 0.333 & -0.125 & 0.445 \\
Controlled-In control & -0.111 & 0.250 & 0.777 \\
Distracted-Focused & 0.443 & 0.500 & 0.555 \\
\hline
\end{tabular}

what they needed. On the other end, those with high profiles felt "it was easy and quite simple to gather the information".

\section{Discussion}

Regarding the assessment of AEBs, both in terms of efficiency and effectiveness, we have obtained results that demonstrate the advantage of profiled exhibition (i.e. profile/non-adaptive test conditions). We have also obtained results that point towards the possible advantage of the adaptability (i.e. profile/adaptive), although the experiment results were non-significant. It is important to highlight the fact these results are for all profiles combined (Figs. 6, 7). If we look at the outcomes of both profiled/non-adaptive and profiled/adaptive test conditions per profile (Figs. 8, 9) we find out that the high priority profile (division director has clear benefits in both the profiling and the adaptation of the presentations.

When looking at the subjective measurements that we made with the bi-polar questionnaires (Sect. 5.3) we gathered results that are not significant. We can only suggest further studies in this area to investigate the affect an adaptive exhibition booth could have on visitors. After each test conducted, participants were offered beverages and invited to comment on the experiment. To our surprise participants made very perceptive comments. In the test condition profile/non-adaptive, most participants felt they had to wait quite a lot for the "loop" (as described by the participants) to come back to some relevant information. Furthermore, some of the design director expressed great frustration in the case of the profile/adaptive test, feeling as if they were low priority. There was expression of frustration by the majority of low profile participants. Some participants guessed that they were being treated as "second class" and could not understand why others would leave the booth earlier. At the same time, those with the top profile felt the experiment was efficient and enjoyable. This is interesting because participants were not told of a ranking or prioritisation of profiles, and it clearly highlights the effects our adaptation strategy has on different user profiles.

During our experiments, participants did talk amongst themselves and did find out that they had different profile. Fortunately they were not able to assess if there was a prioritisation or hierarchy of the profiles. We ensured that participants were not aware of the order of priority given to the different profiles. There is a general acceptance amongst exhibitors and in the literature [e.g. (Terrenghi and Zimmermann 2004)] that booth visitors do not like to be classified or to state what class do they belong to in public. 
There is risk of influence between visitor experiences. In our case, this is particularly true for the 3rd test condition. One example was the satisfaction high profile visitors have while they perceive other visitors as being dissatisfied because of having to wait longer in front of the displays. Symmetrically, the dissatisfaction some visitors experience while they perceive other visitors as being treated better is another example. It is also relevant to remember that visitors influence each other and the expression of satisfaction or dissatisfaction by one visitor will have an effect on the other visitors present.

Another important issue raised in (Masthoff 2004) is the issue of misery. Does satisfaction depend on pleasure only, and in our case in the provision of relevant information to the booth visitors? Or is satisfaction also affected negatively by disagreeable experience, in our case the waiting time to see the relevant information? After the experiments, participants were sensitive to the perception of being treated unfairly and it emerged as a disagreeable experience.

Unlike most systems reviewed (see Sect. 2.4) the one we propose deals with situations when a conflict of resources occurs. That is when visitors with different profiles are simultaneously requesting the same resource (for example, all looking at the same display). What our study has shown is that visitors are very sensitive to the way others are treated and if they feel they are not treated equally they will express strong criticisms. This was all too evident during our post-experiment discussions. During these discussions most participants talked about their experience how they liked or disliked it and how they felt in relation to others. It therefore highlight the surprising fact that affect is at least as important as efficiency and effectiveness in the eyes of the booth visitors.

\section{Conclusion}

Through our experiments and the selection of three test conditions we have demonstrated that profiled non-adaptive presentations are better suited, compared to a generic presentation for an effective and efficient information display strategy. We have demonstrated that the improvement is significant and measurable. We have also demonstrated that the use of profiled and adaptive presentations is promising as a whole and across profiles. While we could not measure a significant objective improvement compared to profile non-adaptive presentations, we have shown that it yields no-worst results. At the same time we have shown that profiled adaptive presentations yield better subjective measurements than either non-profiled non-adaptive presentations or profiled non-adaptive presentations. In the experiment we have set up we were able to gather data from a realistic (in the sense of experiments are based on a realistic simulation of an exhibition booth and test subjects had some freedom regarding their behaviour), settings with all the drawbacks this might have (we notably observed the wandering of visitors in the booth and some of them having short conversations).

Profiled, adaptive presentations as part of an AEB are the direction to follow, because they yield the best combination of objective and subjective results. In other words, when requiring efficiency, effectiveness and positive affect, the profiled adaptive presentations are better suited. Our experiments show that significant room for improvement exist however the results are not significant in highlighting the advantage of adaptation in relation to effectiveness and efficiency, which indicate the necessity of some further investigation of adaptive booths. In particular we should look at the adaptation strategies to implement. We have implemented the "most respected person/dictatorship" strategy, as it is the closest to current industry practice, there is a variety of strategies (see introduction), and we propose to look at these and investigate and implement some of them.

Our contribution was in the architecture and delivery of information within a booth. Our suggestion to use profiles as a means to tailor the information rendered was easily accepted by our industrial partners.

In future work we recommend to investigate the information architecture, the media and the modalities used for an AEB. Further investigations of the effect of AEB on visitors affect are needed, in particular the influence this might have on the visitors memories and experiences of the exhibition. Additionally, assessing and measuring the minimum amount of information seen by each visitor could be used as a measurement of efficiency similar to the proposed measurement of minimum number of pages accessed in a website (Yen and Komg 2002).

Acknowledgment We would like to acknowledge the long-term help from Gielissen (in particular Marc HOYNG) and Private Label Manufacturers Association (PLMA) (in particular, Jan van LIER) for their valuable support and assistance during the execution of this project. The authors wish to thank all the participants and the students who helped with the running of the experiments, Joep KALTHOF and Jan HOEFNAGELS.

Open Access This article is distributed under the terms of the Creative Commons Attribution Noncommercial License which permits any noncommercial use, distribution, and reproduction in any medium, provided the original author(s) and source are credited.

\section{References}

Amato G, Straccia U (1999) User profile modeling and applications to digital libraries. In: Abiteboul S, Vercoustre A-M (eds) ECDL '99, LNCS 1696, Paris, France, pp 184-197 
Banting PM, Blenkhorn DL (1974) The role of industrial trade shows. Ind Mark Manag 3:285-295

Bartneck C, Masuoka A, Takahashi T, Fukaya T (2007) An electronic museum guide in real use. Psychol Aesthet Creativity Arts 1(2):114-120

Bellizzi JA, Lipps DJ (1984) Managerial guidelines for trade show effectiveness. Ind Mark Manag 13:49-52

Bello DC (1992) Industrial buyer behavior at trade shows: implications for selling effectiveness. J Bus Res 25:59-80

Bello DC, Lohtia R (1993) Improving trade show effectiveness by analyzing attendees. Ind Mark Manag 22:311-318

Bieber G, Giersich M (2001) Personal mobile navigation systemsdesign considerations and experiences. Comput Graph 25(4): 563-570

Bullivant L (2006) Responsive environments: architecture art and design. V \& A Contemporary, London, UK

Cavanaugh S (1976) Setting objectives and evaluating the effectiveness of trade show exhibits. J Mark 40(4):100-103

Fan W, Gordon MD, Pathak P (2005) Effective profiling of consumer information retrieval needs: a unified framework and empirical comparison. Decis Support Syst 40:213-233

Goel M, Sarkar S (2002) Web site personalization using user profile information. In: De Bra P, Brusilovsky P, Conejo R (eds) AH 2002, LNCS 2347, Malaga, Spain, pp 510-513

Gopalakrishna S, Lilien GL (1995) A three-stage model of industrial trade show performance. Mark Sci 40(1):22-42

Herbig P, O'Hara B, Palumbo F (1994) Measuring trade show effectiveness: an effective exercise? Ind Mark Manag 23:165-170

Hoshen N (1989) Meeting the right visitors on your stand. Int Trade Forum 25, pp 14-17, 33-34

Korfhage RR (1984) Query enhancement by user profiles. In: Joint BCS and ACM 1984 symposium on research and development in information retrieval, Cambridge, UK, pp 111-121

Kerin RA, Cron WL (1987) Assessing trade show functions and performance: an exploratory study. J Mark 51(3):87-94

Kraemer R, Schwander P (2001) Bluetooth based wireless internet applications for indoor hot spots: experience of a successful experiment during CeBIT. Comput Netw 41(2003):303-312

Krueger MW (1983) Artificial reality. Addison-Wesley, Reading, MA, USA

Liu H, Rauterberg M (2007) Context-aware in-flight entertainment system, part X. Posters at HCI International, Beijing, China, pp $1249-1254$

O'Conner M, Cosley D, Konstan JA, Riedl J (2001) PolyLens: a recommender system for groups of users. In: ECSCW 2001, Bonn, Germany, pp 199-218

Masthoff J (2004) Group modeling: selecting a sequence of television items to suit a group of viewers. User Model User Adapt Interact 14:37-85
McCarthy J, Anagnost T (1998) MusicFX: an arbiter of group preferences for computer supported collaborative workouts. In: CSCW'98, Seattle, WA, USA, pp 363-372

Munuera JL, Ruiz S (1999) Trade fairs as services: a look at visitors' objectives in Spain. J Bus Res 44:17-24

Oppermann R (2005) From user-adaptive to context-adaptive information systems. i-com Zeitschrift für Interaktive und Kooperative Medien 4(3):4-14

Oppermann R, Specht M (1999) A nomadic information system for adaptive exhibition guidance. In: ICHIM99, international cultural heritage meeting, Washington, DC, 1999

Ostrom A, Iacobucci D (1995) Consumer trade-offs and the evaluation of services. J Mark 59:17-28

Pateli AG, Spinellis DD, Giaglis GM (2004) 'Wireless info-communication and navigation services in exhibition shows. In: MBusiness 2004, New York, NY, USA, pp 199-210

Lukose RJ, Adar E, Tyler JR, Sengupta C (2003) SHOCK: communicating with computational messages and automatic private profiles. In: Proceedings of WWW 2003, Budapest, Hungary, pp 291-300

Rosson PJ, Rolf Seringhaus FH (1995) Visitor and exhibitor interaction at industrial trade fairs. J Bus Res 32:81-90

Salem B, Rauterberg M (2004) Multiple user profile merging (MUPE): key challenges for aware environments. In: EU- SAI 2004, Eindhoven, The Netherlands, pp 196-206

Sashi CM, Peretty J (1992) Do trade shows provide value? Ind Mark Manag 21:249-255

Spiegel (1992) Messen und messebesucher in deutchland, Fach and Wissen Band 9, Verlag, Hamburg, as quoted in (Rosson 1995 [29])

Stock O, Zancanaro M, Busetta P, Callaway C, Kruger A, Kruppa M, Kuflik T, Not E, Rocchi C (2007) Adaptive, intelligent presentation of information for the museum visitor in PEACH. User Model User Adapt Interact 17:257-304

Tanner JF, Chonko LB (1995) Trade show objectives, management, and staffing practices. Ind Mark Manag 24:257-264

Terrenghi L, Zimmermann A (2004) Tailored audio augmented environments for museums. In: IUI'04, Madeira, Funchal, Portugal, pp 334-336

Wang Y-F, Chuang Y-L, Hsu MH, Keh H-C (2004) A personalised recommender system for the cosmetic business. Expert Syst Appl 26:427-434

Williams JD, Gopalakrisna S, Cox JM (1993) Trade show guidelines for smaller firms. Ind Mark Manag 22:265-275

Yen BP-C, Komg RCW (2002) Personalisation of information access for electronic catalogues on the web. Electron Commer Res Appl $1: 20-40$ 